\title{
Contrast-Enhanced Cerebral MR Venography Overcomes Causes of Drop of Signal in Time of Flight MR Venography
}

\author{
FATMA M. SHERIF, M.D. and TAMER M.E. BELAL, M.D. \\ The Departments Radiology and Neurology, Faculty of Medicine, Mansoura University, Mansoura, Egypt
}

\begin{abstract}
Background: Cerebral venous thrombosis is uncommon disease with vague manifestations. Magnetic resonance venography (MRV) plays an important role in its diagnosis. The cardinal MRV sign of venous sinus thrombosis is sinus drop of signal. Using time of flight MR venography (TOF MRV) -the commonly used technique there are multiple causes of signal drop simulating dural sinus thrombosis.
\end{abstract}

Aim of Study: To diagnose different causes of signal drop on TOF MRV using contrast enhanced MRV to avoid misinterpretation of dural sinus thrombosis.

Patients and Methods: 79 patients with clinical suspicion of cerebral venous sinus thrombosis underwent cerebral 2DTOF and post-contrast MRV (time-resolved imaging of contrast kinetics (TRICKS) MRV in 40 patients \& elliptic centric MRV in 39 patients). Findings in TOF MRV were compared to those in contrast-enhanced MRV.

Results: Dural sinus thrombosis was seen in 30 cases. Turbulence of flow in superior sagittal sinus and lateral sinuses was demonstrated in 8 cases, hypoplastic transverse sinus was diagnosed in 23 cases, atretic transverse sinus was shown in 2 cases, stenosis of the transverse sinus was seen in 7 cases. 10 cases demonstrated normal dural sinuses on all used MRV techniques.

Conclusion: Drop of signal in dural venous sinuses using TOF MRV has many causes other than thrombosis. To avoid misinterpretation it is important to use contrast enhanced MRV either TRICKS or elliptic centric techniques.

Key Words: Dural sinus thrombosis - TOF MR venography, Time resolved MRV - Elliptic centric MRVHypoplastic transverse sinus.

\section{Introduction}

CEREBRAL venous thrombosis is an uncommon cause of stroke. Usually, patients present with nonspecific symptoms. Prompt diagnosis of CVT is important, as timely and appropriate treatment can reverse the disease process and significantly

Correspondence to: Dr. Fatma Mohamed Sherif, E-Mail: fatema sherif@yahoo.com. reduce the risk of acute and long-term complications [1-3]

Magnetic resonance (MR) neuroimaging is an established method for early diagnosis of cerebral venous thrombosis. However, the sensitivity of non-enhanced MR images is reduced due to a considerable variation of the signal intensity of venous thrombi on $\mathrm{T} 1$ and $\mathrm{T} 2$ weighted images, as well as GRE images according to the thrombus age [4]. Thus, MRV is as necessary for accurate diagnosis [1]

Time of flight MRV is commonly used technique. However, as it is based on flow-related enhancement, a lack of normal venous flow signal intensity is considered positive for thrombosis yielding sometimes to false positive results.

Aim of the work:

The goal of this study was to diagnose different causes of drop of signal on 2-D TOF MRV using contrast enhanced MRV as gold standard technique to avoid false positive results of this technique.

\section{Patients and Methods}

This study was conducted in radiology center from January 2015 till December 2016. 79 patients were studied (mean age $\pm S D=33 \pm 12$ years), 48 females and 31 males, referred from neurology

\footnotetext{
Abbreviations:

MRV : Magnetic resonance venography.

TOF MRV : Time of flight magnetic resonance venography.

TRICKS : Time resolved imaging of contrast kinetics.

DSA : Digital subtraction angiography.

CTA : CT angiography.

FLAIR : Fluid attenuation inversion.

TR : Repetition time.

TE : Echo time

FA : Flip angle

CVST : Cerebral venous sinus thrombosis.
} 
and neurosurgery clinics for clinical suspicion of dural venous sinus thrombosis.

MR examinations were performed on a $1.5 \mathrm{~T}$ 16-channel MR scanner (GE Health care H.D., Milwaukee, Wis) by using a standard head coil. Patients underwent the following sequences in the stated order: T1 weighted images, T2 weighted images, and FLAIR images.

MRV was performed with 2D TOF MRV followed by post-contrast MRV. The parameters used for 2D TOF MRV sequence were as follows: TR/TE, 18/3.1 msec; FA, 20; 40; section thickness, $1.4 \mathrm{~mm}$; FOV, 220mm; acquisition matrix, $256 \mathrm{x}$ 160 and acquisition in coronal image plane.

The post-contrast MRV study was done using (Time Resolved MR angiographic technique) TRICKS in 40 patients \& Elliptic Centric in 39 patients.

The parameters used for the 3D TRICKS sequence were as follows: TR/TE, 36/1.4 msec; FA, 30; section thickness, $1.4 \mathrm{~mm}$; acquisition matrix, 256x 160; TA, 2 minutes; and acquisition in coronal image plane.

The parameters used for the 3D Elliptic centric MRV sequence were as follows: TR/TE, 5.8/2 msec; FA, 40; section thickness, $2.4 \mathrm{~mm}$; acquisition matrix, 256x160; TA, 2 minutes; and acquisition in coronal image plane.

An intravenous gadolinium-based contrast agent was injected at a rate of $2 \mathrm{ml} / \mathrm{sec}$ by using a pressure injector, followed by a $20-\mathrm{ml}$ saline flush. The total volume of contrast agent was $10 \mathrm{ml}$.

MRI and MRV images of all patients were reviewed independently by 2 experienced radiologists. On TOF MRV images, a lack of normal venous flow signal intensity was considered positive for venous sinus thrombosis. Then, elliptic centric contrast enhanced and TRICK MRV were analyzed to confirm or rule out the possibility of false results of TOF MRV.

\section{Results}

79 patients with clinical suspicion of dural venous sinus thrombosis were studied with MRI, TOF MRV followed by post-contrast MRV, elliptic centric MRV (39 patients) and TRICKS MRV (40 patients). As demonstrated in Table (1), 69 cases showed drop of signal in different dural venous sinuses on TOF MRV examination. 30 cases were diagnosed to have dural sinus thrombosis on all performed MRV modalities (Figs. 1,2). With contrast-enhanced MRV, 4 cases showed turbulence of flow in posterior part of superior sagittal sinus (Fig. 3). 3 cases demonstrated turbulent flow in lateral sinus (Figs. 4,6). 23 cases proved to have transverse sinus hypoplasia (Fig. 5), unilateral in 22 cases \& bilateral in one case. Transverse sinus stenosis was depicted in 7 cases (Fig. 6). 2 cases demonstrated transvers sinus atresia/aplasia. Ten cases showed no drop of signal on all MRV techniques.

Table (1): Results of drop signal in dural venous sinuses on 2D TOF MRV and its causes according to findings on contrast-enhanced MRV.

\begin{tabular}{lcc}
\hline Causes of drop of signal on 2-D TOF & Number & Percent \% \\
\hline Dural sinus thrombosis & 30 & 43.5 \\
Turbulence flow in posterior part of superior sagittal sinus. & 4 & 5.8 \\
Turbulence of flow in transverse or sigmoid sinus & 3 & 4.3 \\
Hypoplastic transverse dural sinus: & 22 & 33.3 \\
$\quad$ Unilateral & 1 & \\
Bilateral & 2 & 2.9 \\
Atretic/aplastic transverse sinus unilateral & & 10.1 \\
Stenosis of the transverse sinus: & 6 & \\
$\quad$ Unilateral & 1 & \\
Bilateral & & \\
\hline
\end{tabular}



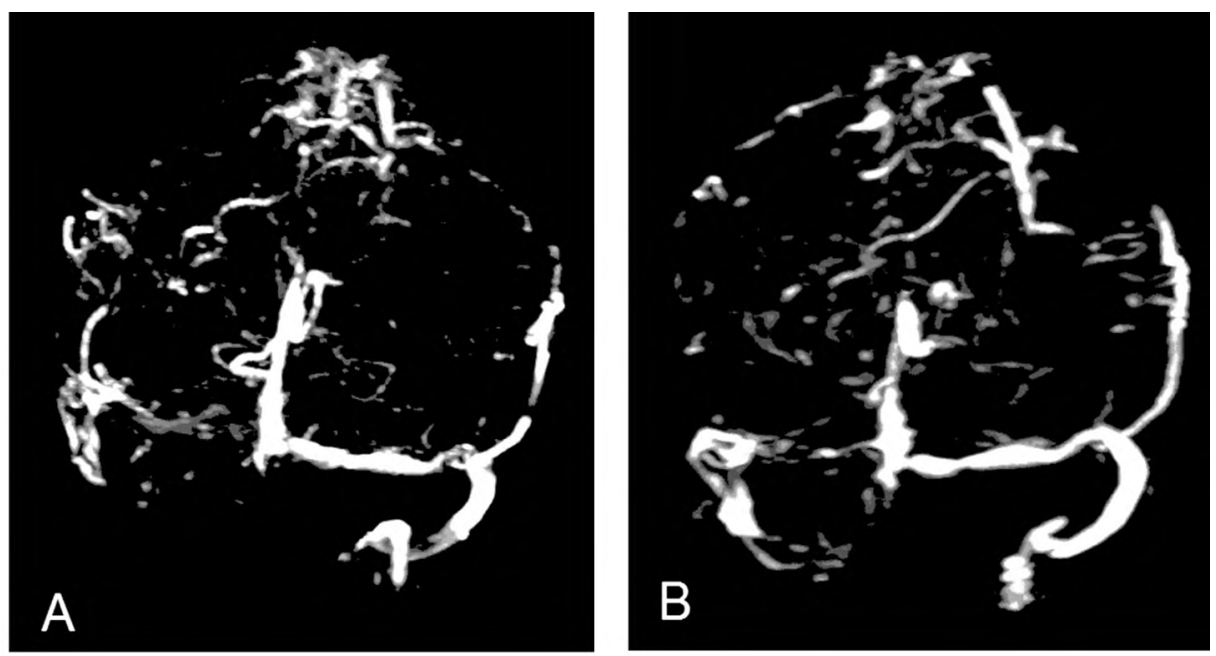

Fig. (1): Male patient 13 years old complaining of papilledema. (A) TOF \& (B) Contrast-enhanced MRV show thrombosis of the superior sagittal and right transverse sinuses.
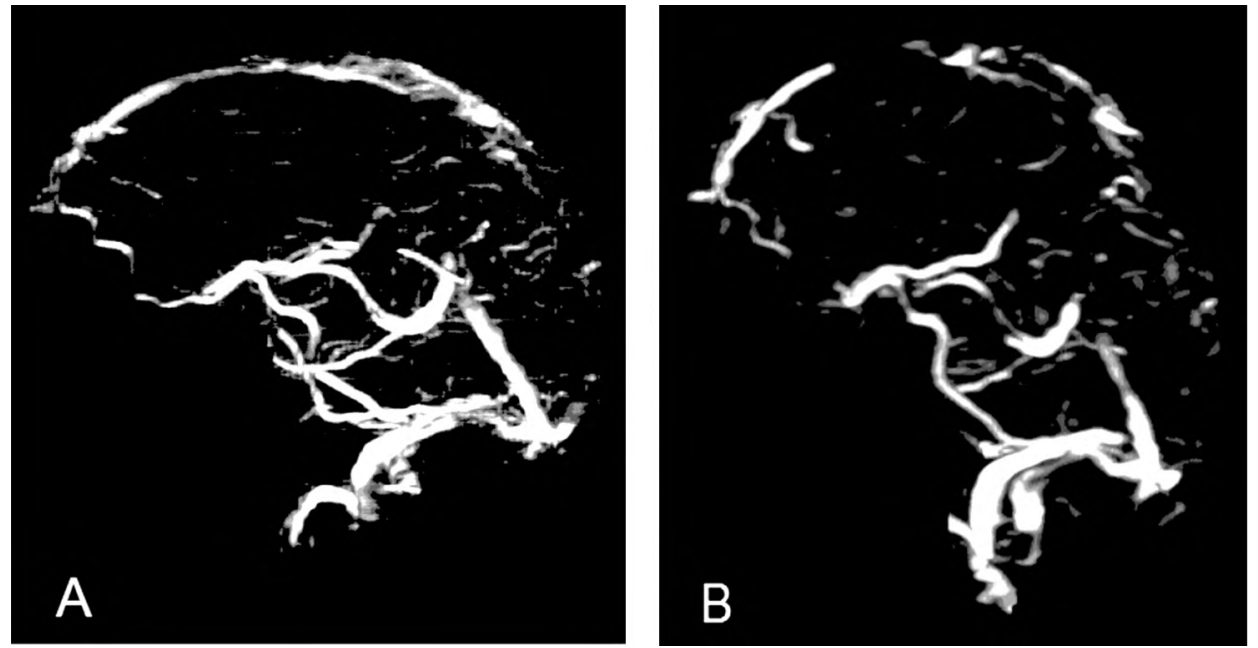

Fig. (2): Female patient 40 years old with impaired consciousness. (A) TOF \& (B) Contrast-enhanced MRV show thrombosis of the superior sagittal sinus.
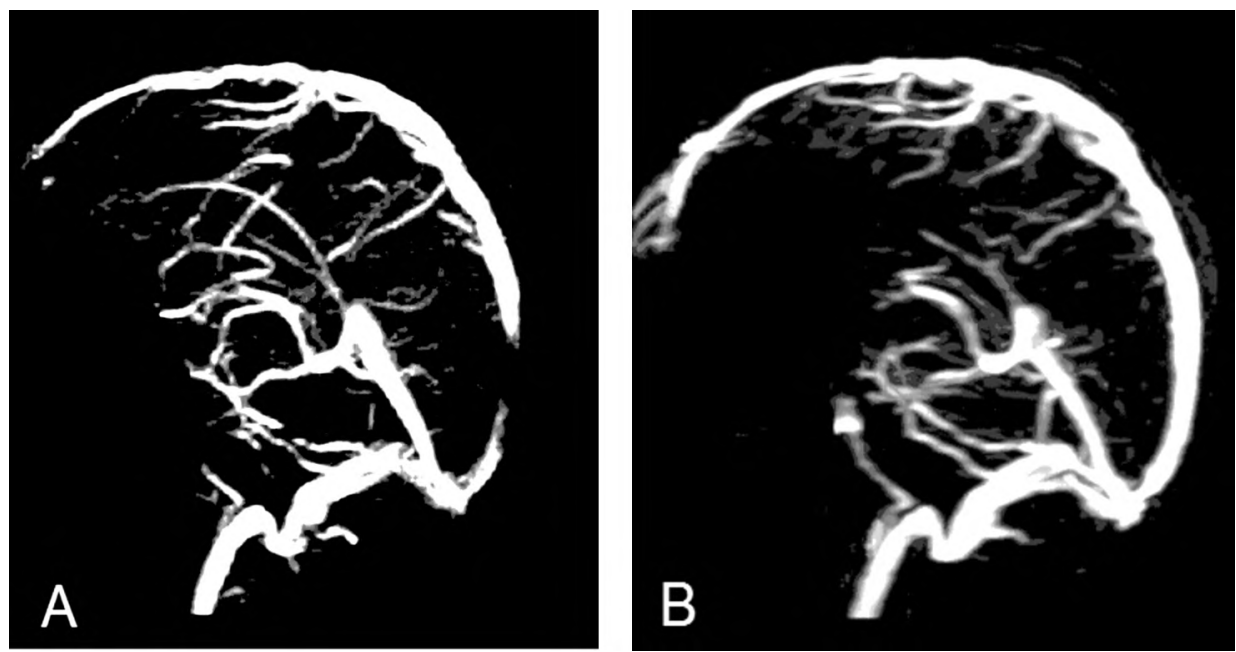

Fig. (3): Female patient 27 years old with sudden onset of severe headache. (A) TOF \& (B) Contrastenhanced MRV: Turbulent flow in posterior part of SSS causing drop of signal in TOF and patent superior sagittal sinus in contrast-enhanced MRV. 

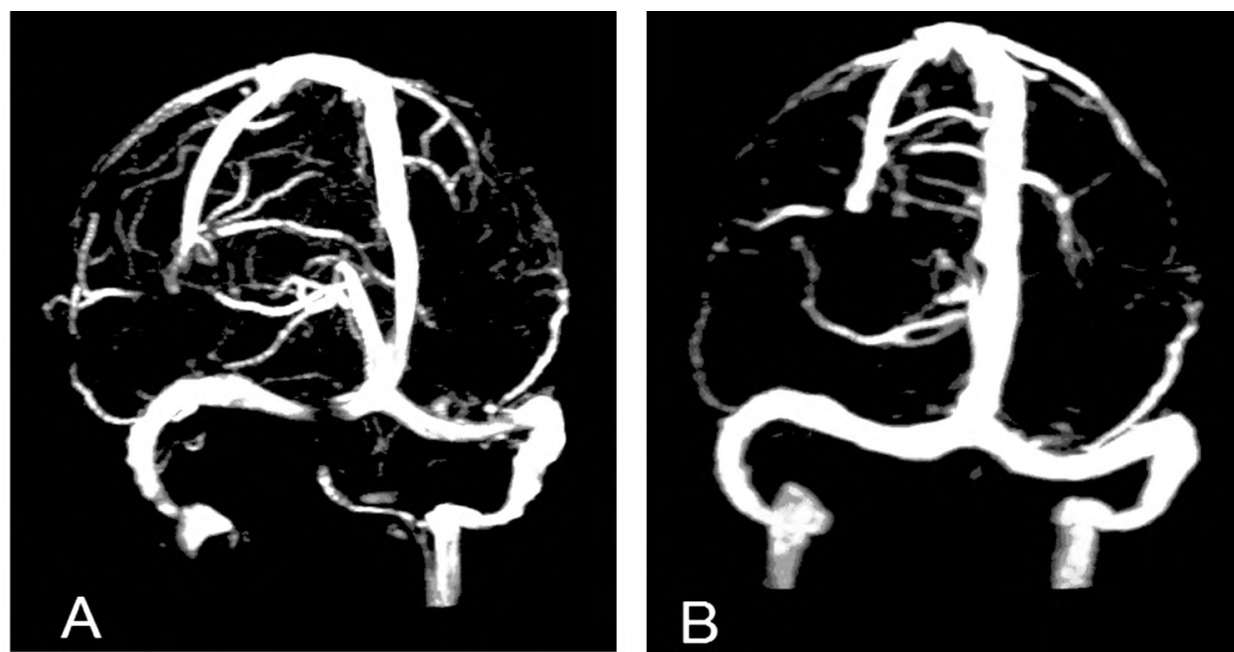

Fig. (4): Male patient 25 years old with severe headache. (A) TOF \& (B) Contrast-enhanced MRV. Drop of signal in the proximal part of right transverse sinus in TOF MRV due to turbulent flow.
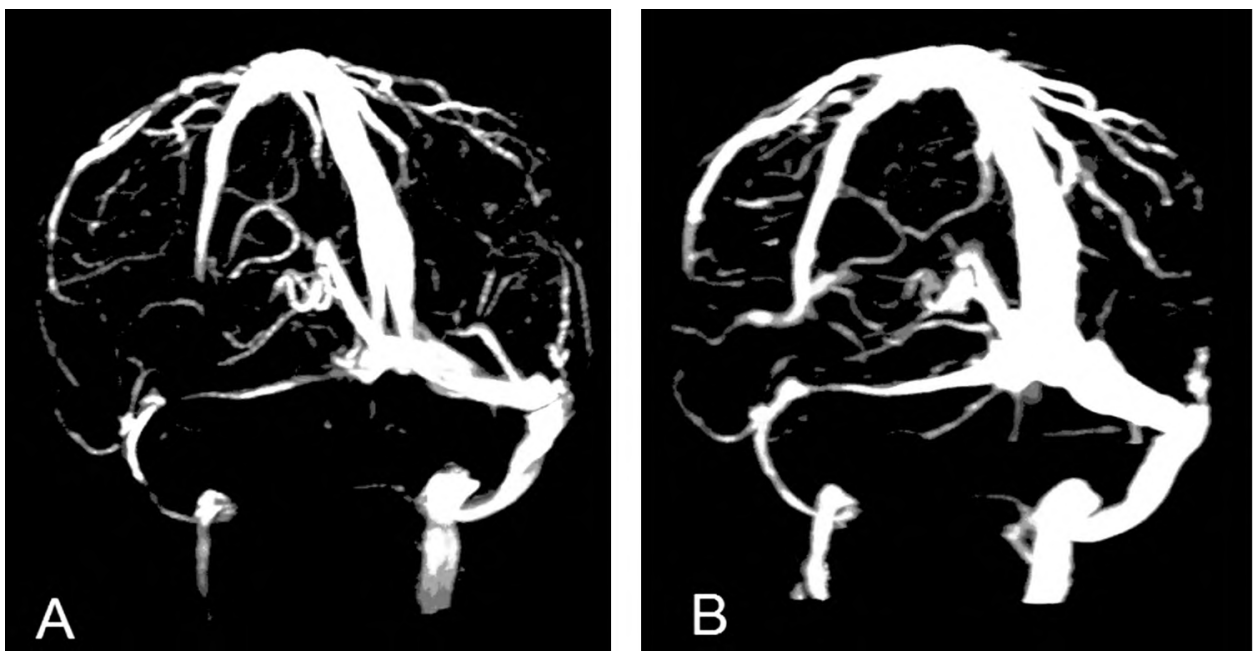

Fig. (5): Male patient 10 years old with disturbed conscious level. (A) TOF \& (B) Contrast-enhanced MRV: Hypoplastic left transverse sinus (as shown in contras-enhanced MRV) causing drop of signal in TOF.
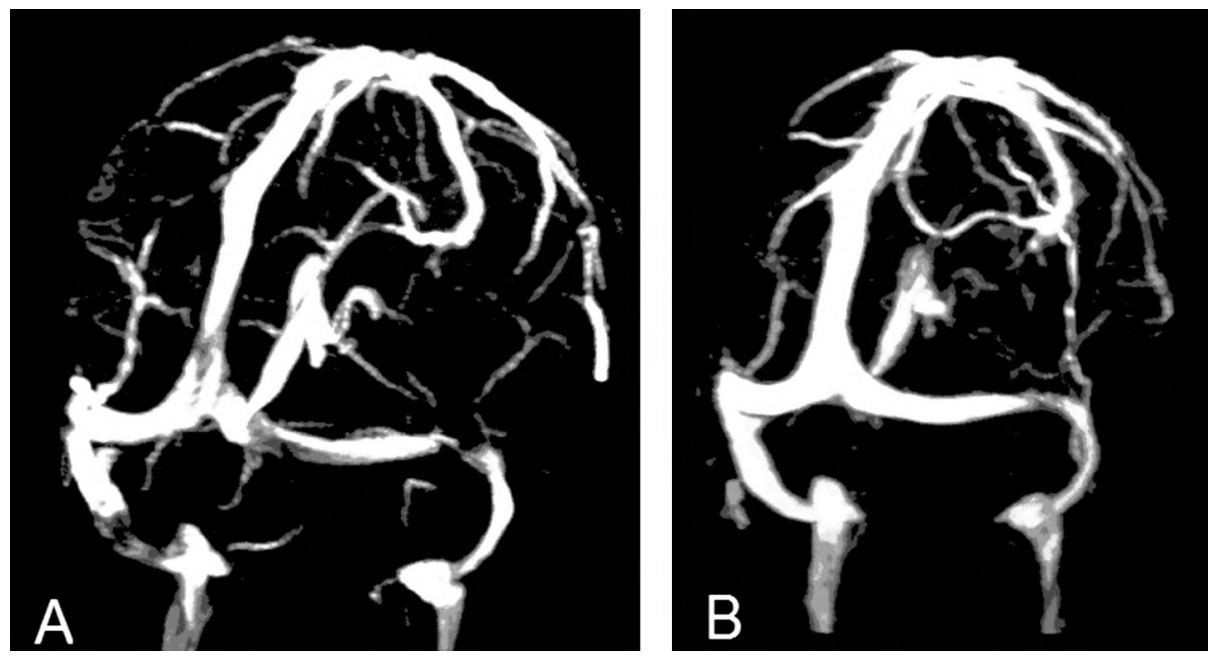

Fig. (6): Female patient 28 years old with seizures. (A) TOF \& (B) Contrast-enhanced MRV: Stenosis of the distal part of right transverse sinus and turbulent flow in the distal part of left sigmoid sinus (as shown in contras-enhanced MRV) causing drop of signal in TOF. 


\section{Discussion}

Cerebral venous sinus thrombosis (CVST) is an uncommon but potentially fatal disease marked by blood clotting in cerebral veins and dural venous sinuses. Its incidence rate accounts for $0.5-2.0 \%$ of strokes and it is sometimes misdiagnosed by clinicians [5]. It was previously related to infections such as mastoiditis. However, due to use of modern effective antibiotics, it is more often related to pregnancy, puerperium, oral contraceptive use, dehydration, intracranial tumors as well as coagulopathies [6,7].

Imaging studies are the cornerstone for diagnosis of CVST. The imaging modality of choice is MR venography as it allows direct visualization of the large cerebral veins and dural venous sinuses. In conjunction with conventional MRI, MRV is reliable as the sole examination for diagnosis of CVST. TOF MRV is the most commonly used technique of examination as it is fast, simple and does not need use of contrast agent. However it probably yields false positive results because flowrelated and susceptibility artifacts can impair the evaluation of the venous structures, it was stated that the sensitivity and specificity of TOF MRV in detection of CVST were $63.6 \%$ and $48.4 \%$ respectively [8,9]. Its main drawbacks include an insensitivity to in-plane flow, patient motion causing vessel misregistration among the slices, and high signal from substances with short $\mathrm{T} 1$ values such as fat and methemoglobin which can mimic flow because of incomplete saturation and "shine through" on the MIP reconstruction. Thus, not all drop of signal in dural venous sinuses means thrombosis [10-12].

Contrast- enhanced MRV takes the advantage of contrast agent luminal filling rather than relying on the MR flow phenomena as in TOF MRV. Recently, gadolinium-enhanced MRV has been demonstrated to be superior to TOF MRV and may offer the best evaluation using MRI. It was declared that for diagnosis of dural venous sinus thrombosis, dynamic contrast-enhanced MRV demonstrated sensitivity of $97 \%$ and specificity $(99 \%) \quad[\mathbf{1 , 1 3}, 14]$

In this study we used two contrast-enhanced (CE) MRV techniques to depict causes of lack of signal in dural venous sinuses shown with TOF MRV.

Among the seventy nine patients of this study who were examined with TOF MRV followed by contrast-enhanced MRV in the same session looking for CVST performed over a two-year period, 30 patients were diagnosed to have dural venous sinus thrombosis and 10 patients had normal study on all performed MRV studies. The rest of examined cases demonstrated drop of signal simulating dural venous sinus thrombosis with TOF MRV examination. However, with contrast-enhanced TRICKS and Elliptic Centric MRV, the results were different; about $33.3 \%$ of cases demonstrated hypoplastic transverse sinuses, either unilateral or bilateral. 7 cases showed transverse sinus stenosis, either unilateral or bilateral. Two cases showed transverse sinus atresia. Also, four cases demonstrated turbulence of flow in the posterior part of superior sagittal sinus and three cases showed transverse/ sigmoid sinus turbulence of flow.

In our study, four cases showed filling defect in the superior sagittal sinus on TOF MRV, while on contrast-enhanced MRV, normal filling was depicted. This False-positive finding on TOF MRV could be attributed to use of inferior saturation band used to saturate arterial signal on using source images in the axial plane [7].

Also, anatomical causes of false-positive findings for CVST on MRV can be due to variations in the size of the dural sinuses and anatomic structures producing filling defects. An asymmetric appearance because of hypoplasia or aplasia of one of the transverse sinuses is noticed in up to $25 \%$ of healthy patients on TOF MRV reflecting that seen at catheter angiography [15]. This was demonstrated in our study as $33.3 \%$ of cases showed hypoplastic transverse sinuses on contrastenhanced MRV, either unilateral or bilateral. This is also in agreement with Goyal et al., 2016 who stated that transverse sinus hypoplasia is the most common anatomical variant of cerebral dural venous sinus anatomy [16].

However, in some cases, the discrepant size of the transverse sinuses could be misdiagnosed as thrombosis [7]. This may be augmented when a small sinus is viewed on maximum-intensityprojection (MIP) images, especially in infants, denoting that the evaluation of source images when a sinus is not initially visualized on MIP images [17].

In conclusion, not all drop of signal in dural venous sinuses with TOF MRV should be considered thrombosis. Contrast-enhanced MRV study can be better used for more precise evaluation of the dural venous sinuses.

\section{References}

1- MECKEL S., REISINGER C. and BREMERICH J.: Cerebral venous thrombosis: Diagnostic accuracy of combined, dynamic and static, contrast-enhanced 4D MR 
venography. AJNR American Journal of Neuroradiology, 31: 527-35, 2010.

2- IQBAL N. and SHARMA A.: Cerebral Venous Thrombosis: A mimic of brain metastases in colorectal cancer associated with a better prognosis. Case reports in oncological medicine, article ID 109412, 3 pages, 2013.

3- ZHU X. and CAO L.: Cerebral Venous Sinus Thrombosis following diagnostic curettage in a patient with uterine fibroid. Case Reports in Neurological Medicine, 270654: $1-4,2014$

4- LEACH J.L., STRUB W.M. and GASKILL-SHIPLEY M.F.: Cerebral venous thrombus signal intensity and susceptibility effects on gradient recalled-echo MR imaging. AJNR American Journal of Neuroradiology, 28: 94045, 2007.

5- QU H. and YUNG M.: Early imaging characteristics of 62 cases of cerebral venous sinus thrombosis. Experimental and Therapeutic Medicine, 5: 233-236, 2013.

6- POON C., CHANG J., SWARNKAR A., et al: Radiologic diagnosis of cerebral venous thrombosis: Pictorial review. American Journal of Radiology, 189: S64-S75, 2007.

7- PROVENZALE J. and KRANZ P.: Dural sinus thrombosis: sources of error in image interpretation. AJR American Journal of Radiology, 196: 23-31, 2011.

8- KLINGEBIEL R., BAUKNECHT H., BOHNER G., et al.: Comparative evaluation of 2D time-of-flight and 3D elliptic centric contrast-enhanced MR venography in patients with presumptive cerebral venous and sinus thrombosis. European Journal of Neurology, 14: 139-143, 2007.

9- FU J., LAI P., HSIAO C., et al: Comparison of real-time three-dimensional gadolinium-enhanced elliptic centricordered MR venography and two-dimensional time-of- flight MR venography of the intracranial venous system. Journal of Chinese Medical Association, 73: 131-138, 2010 .

10- SCOTT J. and FARB R.: Imaging and anatomy of the normal intracranial venous system. Neuroimaging Clinics of North America, 13: 1-12, 2003.

11-AYANZEN R., BIRD C., KELLER P., et al: Cerebral MR venography: Normal anatomy and potential diagnostic pitfalls. AJNR American Journal of Neuroradiology, 21: 74-78, 2000

12- PAOLETTI M., GERMANI G., De ICCO R. et al: Intraand extracranial MR venography: Techniqual notes, imaging application and imaging development. Behavioral Neurology, 2694504: 1-9, 2016.

13- CHIEWVIT P., PIYAPITTAYANAN S. and POUNGVARIN N.: Cerebral venous thrombosis: Diagnosis dilemma. Neurology International, 3: e13: 50-56, 2011.

14- HU H. HAIDER C., CAMPEAU N., HUSTON III J. and RIEDERER S.: Intracranial contrast-enhanced magnetic resonance venography with 6.4-fold sensitivity encoding at 1.5 and 3.0 tesla. Journal of Magnetic Resonance Imaging, 27 (3): 653-658, 2009.

15- ALPER F., KANTARCI M., DANE S., GUMUSTEKIN K., ONBAS O. and DURUR I.: Importance of anatomical asymmetries of transverse sinuses: An MR venographic study. Cerebrovascular Diseases, 18: 236-239, 2004.

16- GOYAL G., SINGH R., BANSAL N. and PALIWAL V.: Anatomical variations of cerebral MR venography: Is gender matter? Neurointervention, 11: 92-98, 2016.

17- ROLLINS N., ISON C., BOOTH T. and CHIA J.: MR venography in the pediatric patient. AJNR American Journal of Neuroradiology, 26: 50-55, 2005.

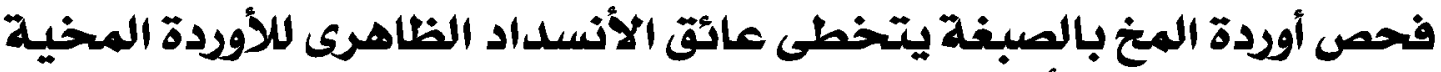 بفحص أوردة الهخ بلدون صبغة بطقيقيقة زمن الطيران}

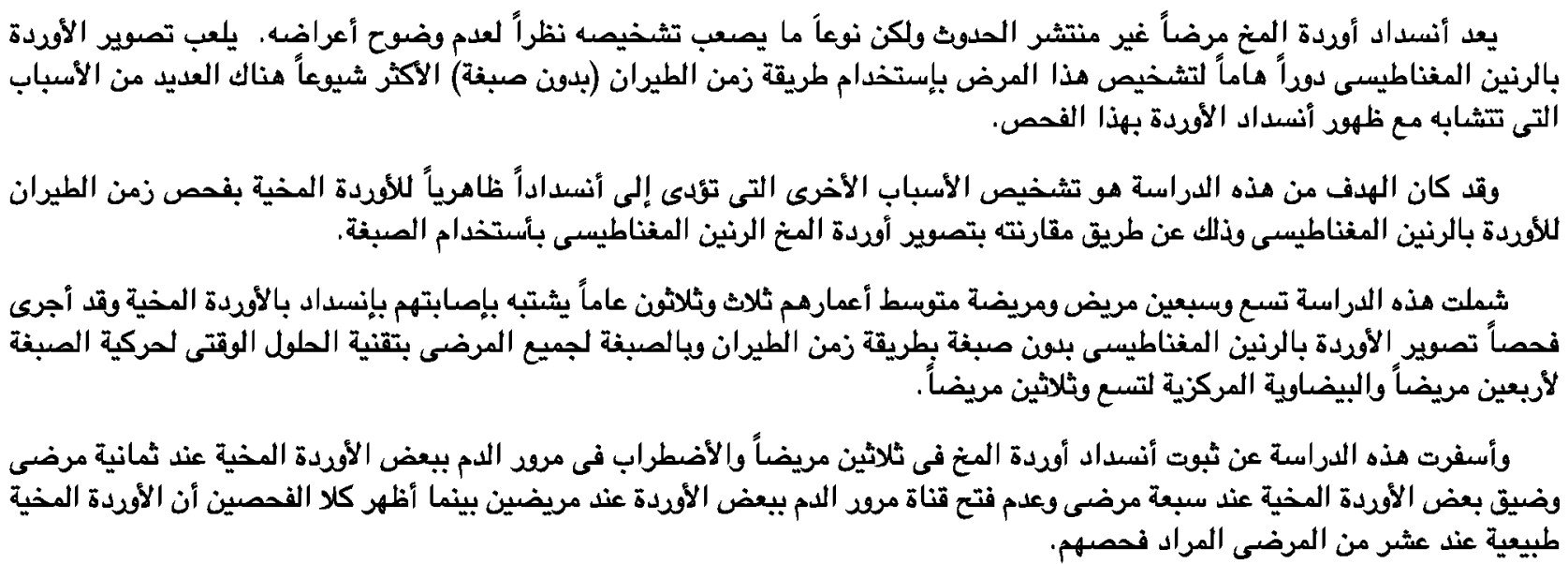

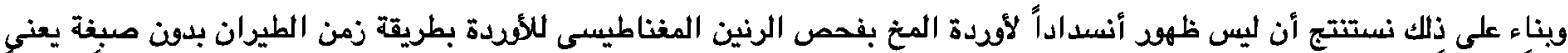

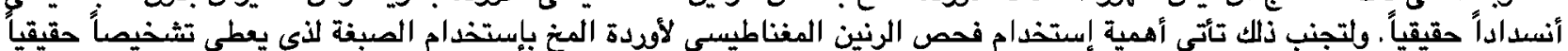

